\title{
SOCIAL MEDIA AS AN OPPORTUNITY FOR PUBLIC HEALTH INTERVENTIONS: THE \#METOO MOVEMENT AS AN EXEMPLAR
}

\author{
Raquel Gómez Bravo MD¹, María Gómez Bravo², Charilaos Lygidakis MD¹, Claus Vögele PhD ${ }^{1}$ \\ ${ }^{1}$ Institute for Health and Behaviour. Research Unit INSIDE. University of Luxembourg \\ ${ }^{2}$ Sra. Rushmore United (WPP Group), Madrid, Spain
}

\begin{abstract}
Background: Social media have been used exponentially and globally, providing a means for billions of users to connect, interact, share opinions and criticise, becoming one of the main channels of communication for users around the world. One of the most popular free social media networks is Twitter, with more than 100 million active users per day worldwide. Purpose: The aim of this study was to analyse a sample of the public conversations generated, using the hashtag \#MeToo, around the topic of sexual abuse on Twitter. Methods: Using social media marketing software, the use of the \#MeToo hashtag was analysed over a period of 60 days (14 September 2017 to 13 November of 2017). Results: The \#MeToo conversation was mainly in English (79.3\%), located in the United States (48.2\% of cases), but with global repercussions. The volume of mentions of the \#MeToo hashtag was far greater (97.7\%), compared with other hashtags related to violence over this period of time, using mostly Twitter $(96.2 \%)$. Conclusions: These results suggest that it is possible to describe different groups using the social media, and analyse their conversations to identify opportunities for successful public health interventions. If the topic is relevant for the general public, it will generate interest and conversations at the global level, supported by a universal and borderless channel such as Twitter.
\end{abstract}

Keywords: social media; Twitter; sexual harassment; public health; primary prevention.

Gómez Bravo R, et al. JISfTeH 2019;7:e5(1-7).

DOI: https://doi.org/10.29086/JISfTeH.7.e5

Copyright:@ The Authors 2018

Open access, published under Creative Commons Attribution 4.0 BY International Licence

\section{Introduction}

Social media use has grown exponentially and is used globally, becoming a standard means of communication for many people. ${ }^{1}$ In the medical field, social media have been employed by professionals and researchers as an efficient way for sharing information, networking, building conversations, staying up-to-date and collaborating with peers and patients. ${ }^{2}$ Social media have also been used by patients mainly for social support, but also for emotional expression and social comparison, as shown in a recent systematic review. ${ }^{3}$

Twitter is one of the most popular free social media networks worldwide, widely used for micro-blogging. In a 280-character message ("tweet") users can post photos and videos, mention others, add keywords using the hash symbol before a word ("hashtag"), and re-share ("retweet") messages, thus spreading information to followers. Hashtags make it easier to find and share information related to specific topics, as they facilitate the discovery of messages, the formation of lists of interests, and the engagement of the community. Tweets are posted publicly on the users' profiles, but the network also supports private "direct messages". There are 100 million active users per day around the world on Twitter, ${ }^{4}$ making it one of the most influential social media networks for generating conversation, following the latest news updates, and receiving important communications from state actors and agencies. This is one of the reasons why Twitter has also attracted the interest of healthcare professionals and reasearchers. ${ }^{5}$

Public health is "the science and art of preventing disease, prolonging life and promoting human health through organized efforts and informed choices of society, organizations, public and private, communities and individuals". ${ }^{6}$ Violence against women is considered a global public health problem and a violation of human rights, which seriously affects women's health (physical, sexual, reproductive, and mental health), with a potentially large impact on morbidity and mortality. ${ }^{7}$ According to the WHO, "action is clearly needed", underlining that especially the health sector has an important role to play, considering the serious health risks faced by women and their families. ${ }^{7}$

"Me Too" is a well-known movement against sexual harassment and assault. The phrase was first used in 2006 by the American social activist and advocate Tarana Burke, but 
its use expanded after the sexual misconduct allegations against Harvey Weinstein and others. $^{8}$ In particular, messages containing the \#MeToo hashtag spread rapidly in October 2017, after being popularised by actress Alyssa Milano, who encouraged victims of sexual harassment to tweet about their own experiences and to highlight the magnitude of the problem. Eventually, the use of this hashtag significantly impacted the discourse regarding workplace culture. ${ }^{9}$ In this sense, the \#MeToo movement appears as an opportunity to raise awareness of sexual harassment aa a public health issue. ${ }^{10}$

Media campaigns can directly or indirectly affect healthrelated behaviours across large populations. ${ }^{11}$ They are considered powerful tools in health promotion and disease prevention because of their wide out-reach, appeal, and costeffectiveness. ${ }^{12}$ Therefore, careful planning of the contents of campaigns, and their fit with the target audiences are crucial. $^{11}$ There is evidence for the success of such campaigns to be determined by factors such as targeting, networking, use of SMART (specific, measurable, achievable, realistic, time bound) objectives, continuous evaluation, and compliance with national and international policies. $^{13}$

The social media have become one of the main channels of communication for users around the world. One of the most popular free social media networks is Twitter, with more than 100 million active users per day worldwide. Despite its widespread use by the public and health care professionals, there is little research on patterns of hashtaguse on Twitter $^{14}$ and a shortage of evidence regarding its impact on public health. ${ }^{15-17}$ Investigating messages and behaviour on social media could provide a unique insight into the possible effects of health information reaching large segments of the public. ${ }^{18-19}$ There is a growing body of literature called "Infodemiology", ${ }^{1}$ which includes research such as Eysenbach's pioneering study on the relationship between Internet search behaviour and influenza incidence, ${ }^{18}$ or Cooper and colleagues' work on exploring the relationship between information demand on cancer, (e.g. incidence, mortality), and news coverage (information supply). ${ }^{8,20}$ Eysenbach defines this as the science of distribution in an electronic medium, specifically the Internet, or in a population, with the ultimate aim to inform public health and public policy. ${ }^{18}$

The current study analyses the public conversation concerning the topic of sexual abuse on Twitter articulated around the hashtag \#MeToo using the high-profile example of Harvey Weinstein as an exemplar, and examining the opportunities that such cases offer for impacting public health interventions.

\section{Methods}

Posts in various social media networks were examined over a period of 60 days - from 14 September to 13 November
2017 - using the social listening tool Radian6 (developed by Salesforce), ${ }^{22}$ which collects and analyses information shared publicly online. ${ }^{23}$ This period was selected as it includes the date of the public fall of Harvey Weinstein on 8 October 2017, which propelled the \#MeToo movement and triggered the documentation of the prevalence of sexual assault and harassment in the workplace. The analysis and monitoring criteria were specified in a broad manner, without geographical or linguistic limitations. The main hashtag "\#MeToo" was compared with other specific keywords related to violence against women: \#genderviolence, \#ipv, \#familyviolence, \#childabuse and \#elderabuse.

A topic trend analysis was used to determine the highest and lowest mentioning activity of the hashtag. This module is provided by Radian6 and determines the highs and lows of any brand in terms of mentions. This module allows for the analysis of the number of mentions and the identification of factors contributing to their gain or loss. Such an analysis supports campaign monitoring or crisis management, as it demonstrates the positive or negative impact of a hashtag. ${ }^{23}$ It is also most helpful for keeping track of conversation trends over a period of time and understanding why a conversation had a spike in a specific moment. ${ }^{23}$ Finally, word clouds of the conversations were created, analysing the top 50 words from the last 1,000 messages containing the selected hashtags.

\section{Results}

The mentions of the \#MeToo hashtag were significantly higher $(97.7 \%)$ than other hashtags related to violence (i.e., \#genderviolence, \#ipv, \#familyviolence, \#childabuse, \#elderabuse, \#intimatepartnerviolence). Twitter was used in $96.2 \%$ of the cases; the remaining $4.8 \%$ were distributed amongst other social media networks (comments on any social media post $1.9 \%$, blogs $0.6 \%$, Facebook $0.1 \%$, forum replies $0.5 \%$, mainstream news $0.4 \%$, content aggregators $0.2 \%$, other $0.2 \%$ ).

Of the messages containing the \#MeToo hashtag $79.3 \%$ were in English. $48.2 \%$ of the messages originating from the United States, followed by the UK, Canada, India, the Netherlands and France with respectively 4.7\%, 3.8\%, 3.3\%, $2.7 \%$ and $1.9 \%$ of the messages. As expected, the use of the hashtag in English triggered a conversation around sexual harassment worldwide, with messages and content being posted in the same language.

The results of the trend analysis are presented in figure 1. The peak coincides with the launch of the tweet of Alyssa Milano on $15^{\text {th }}$ October 2017 (Figure 2), which was followed by a multitude of celebrities and users adding messages with denunciations, support and solidarity using the hashtag \#MeToo. Only 24 hours later, that tweet reached almost 50,000 re-tweets.

In comparison with the trend of other hashtags, which remained stable over the same time period (Figure 3), 


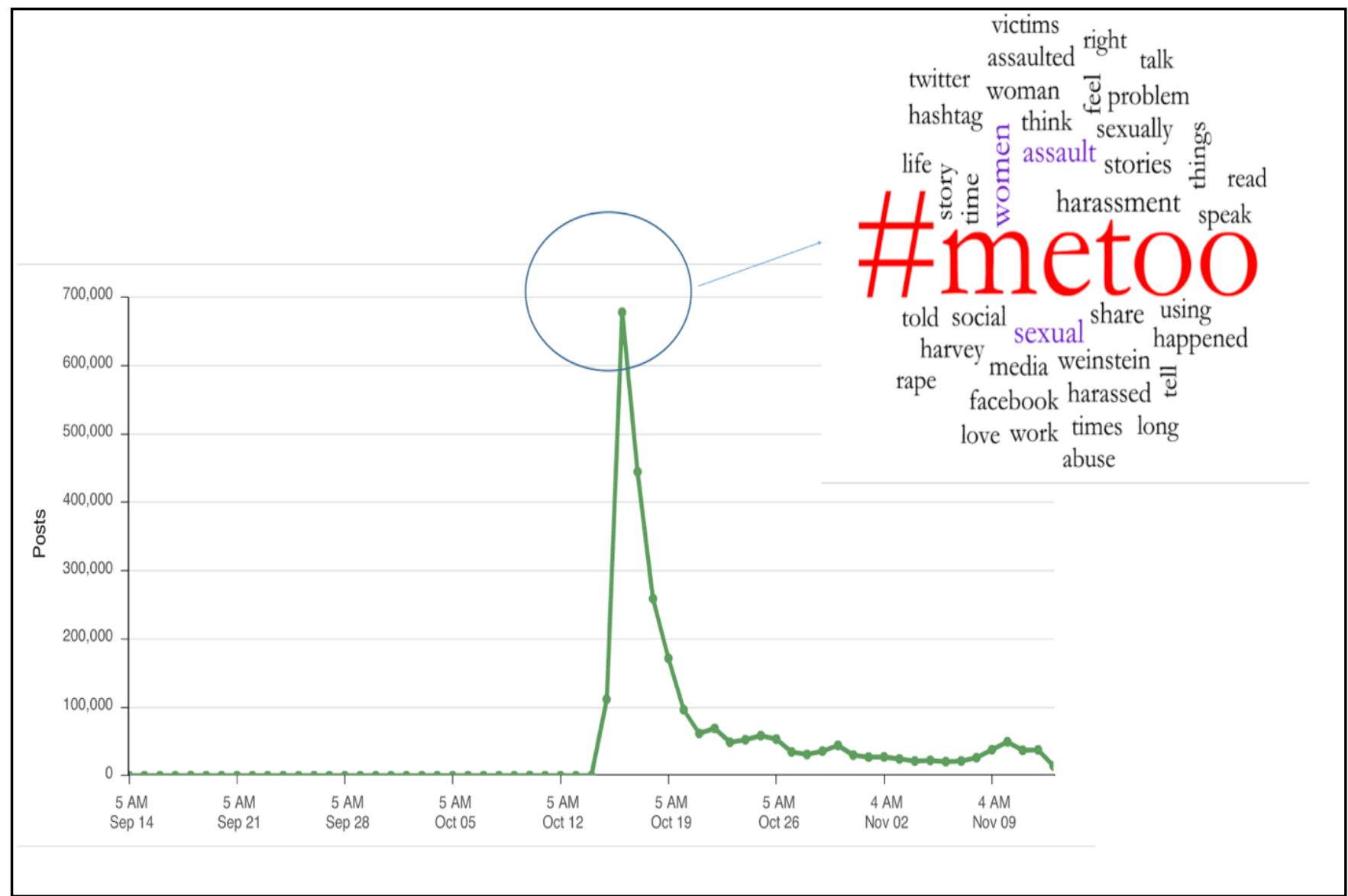

Figure 1. Topic trends analyses of the hashtag \#MeToo.

Me too.

\section{Suggested by a friend: "If all the women who have been sexually harassed or assaulted wrote 'Me too.' as a status, we might give people a sense of the magnitude of the problem."}

\section{ก. Alyssa Milano \\ (9) Alyssa_Milano}

If you've been sexually harassed or assaulted write 'me too' as a reply to this tweet.

22:21 - 15 oct. 2017

○ 52,6 mil 990,8 mil personas están hablando de esto

Figure 2. Tweet of Alyssa Milano (October 15, 2017).

\#MeToo had a noteworthy spread with more than 2.5 million impressions by mid-October. Examples of word clouds, which allowed exploration of the main conversation topics related to the keywords defined in the analysis, are presented in the word clouds of Figures 4, 5 and 6. The keywords employed in the conversations can lead to additional insights.

To explore the impact of the hashtag \#MeToo off-peak, we also selected a random day (6 November 2017) and examined the conversation around \#MeToo. In just 7 minutes, approximately 100 tweets were posted by 84 contributors, reaching more than 274,620 accounts and 443,840 impressions. (Figure 7) For Twitter metrics, 'reach' represents the size of an audience for a conversation, and 'impressions' measures the total number of views of a conversation.

\section{Discussion}

The \#MeToo movement has accomplished an important milestone by opening up an area which was considered by many a taboo. ${ }^{24}$ It is a perfect example of how a discussion on sexual harassment has affected society, raising awareness, ${ }^{25}$ and becoming an opportunity for the public to consider sexual harassment as a health problem with its implications for prevention and promotion. ${ }^{10}$ 


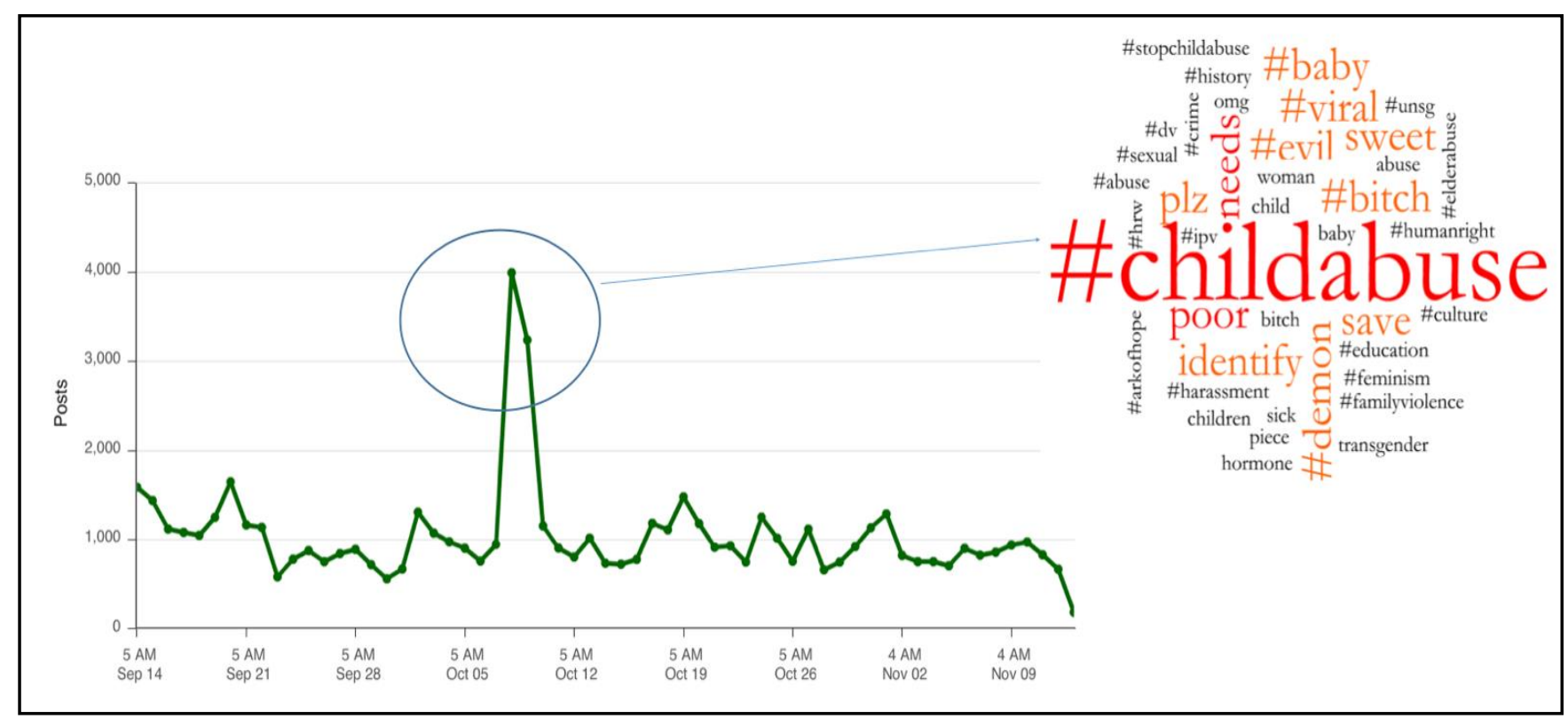

Figure 3: Topic trend analysis and conversation cloud of the hashtag \#childabuse.

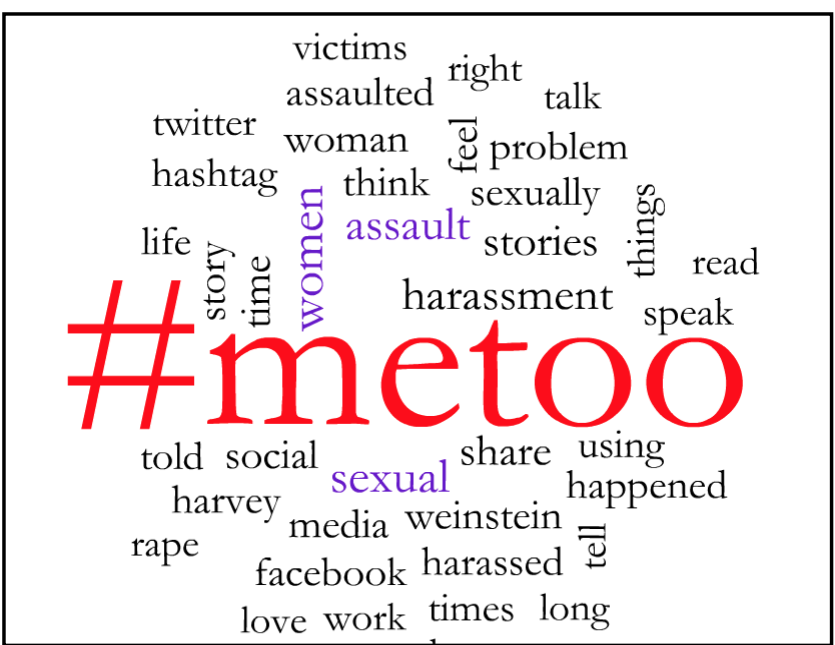

Figure 4. Word cloud for the \#MeToo hashtag.

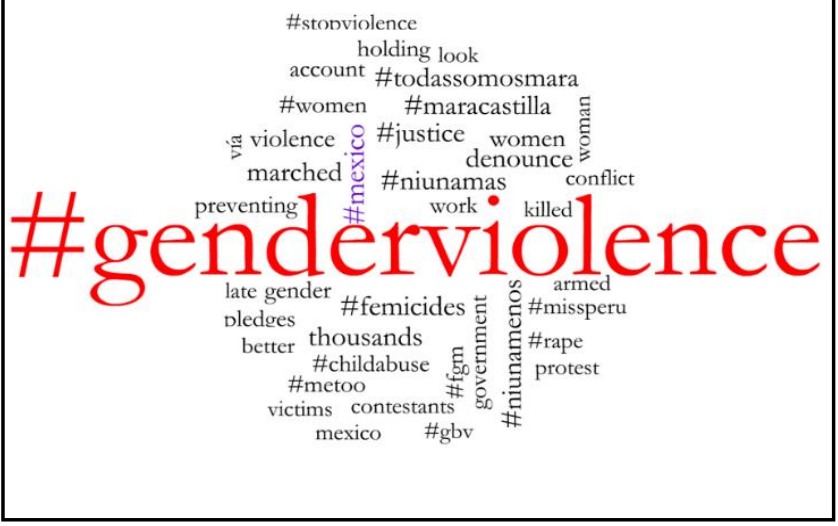

Figure 5. Word cloud for the \#genderviolence hashtag.

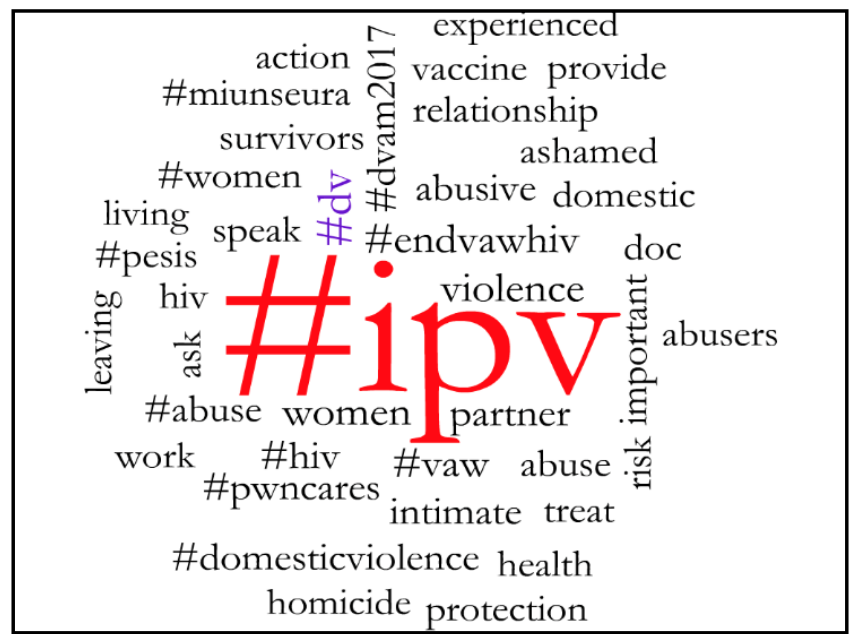

Figure 6. Word cloud of the \#ipv hashtag.

The World Health Organization (WHO) calls for a major scaling up of global efforts to prevent all kinds of violence against women by addressing the social and cultural factors behind it. ${ }^{7}$ This is particularly urgent as violence against women is considered a "global health problem of epidemic proportions" with more than 1 in 3 women being a victim of sexual harassment or gender-based physical or sexual violence, and resulting in serious health and mental health consequences. ${ }^{7}$

Even when women do not label their experiences as "sexual harassment", negative outcomes are evident across lines of the industry sector, occupation, ethnicity, race, and social class. Sexual harassment undermines professional and educational attainment, and mental and physical health. ${ }^{7}$ A 2012 review found that sexual harassment doubled the risk of women undergoing persistent psychological distress two 


\section{\# metoo}
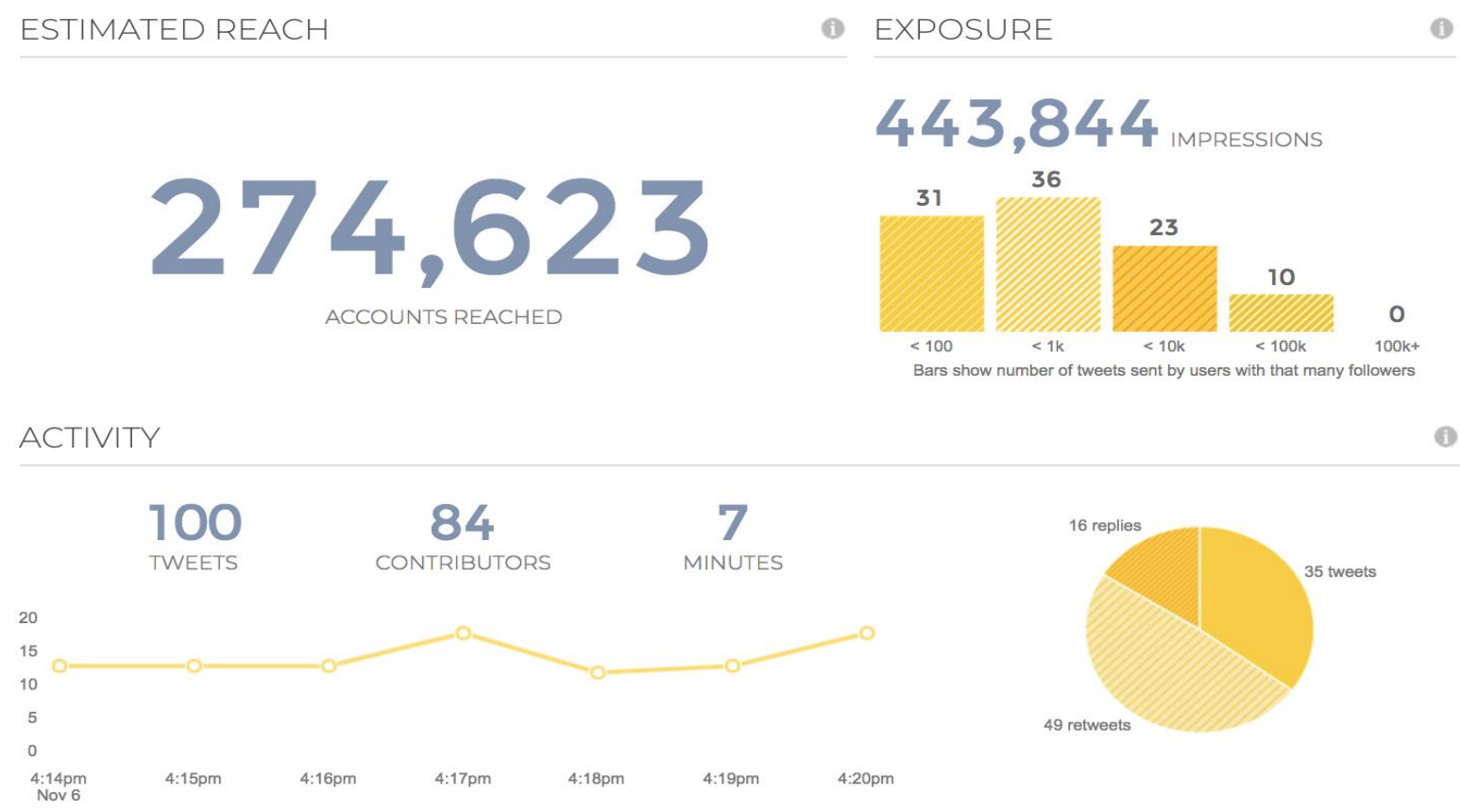

Figure 7. A snapshot of the \#MeToo hashtag metrics on $6^{\text {th }}$ November 2017.

years after their experience, while such a risk was not evident in men. ${ }^{26}$ Any form of gender violence, including sexual harassment, acts as a chronic stressor - even after the removal of the threat. It is well known that for some chronic diseases (including cardiovascular diseases) and cancers, stress is an independent risk factor. ${ }^{27}$

Efforts for primary prevention are increasingly recognised as critical and necessary. Strategies to prevent sexual violence before it occurs include universal interventions directed at the general population, as well as selected interventions aimed at those who may be at increased risk for perpetrating sexual violence. ${ }^{28}$ Not only is there a lack of effective prevention strategies for sexual violence, but there are also gaps in the literature as to whether or not these initiatives to prevent sexual harassment in the workplace impact health outcomes effectively. ${ }^{10,28}$

Social media can be used for media campaigns for health promotion and disease prevention when campaigns are properly planned and targeted to specific groups of population. $^{11}$

This study supports the need to explore the audience of social media and their conversations, analyse the users' interests in different topics, detect the needs and concerns of the population, explore the local and international impact, and, subsequently, design and establish proper successful public health interventions.

Future research should focus on the content of the conversations and interactions, their impact and more importantly, invest in rigorous evaluation research to ensure efficient interventions using social media. Increased societal awareness could help identify target populations and contribute to develop adequate preventive campaigns.

\section{Corresponding author: \\ Raquel Gómez Bravo \\ Institute for Health and Behaviour \\ Research Unit INSIDE \\ University of Luxembourg \\ eMail: raquel.gomezbravo@uni.lu}

Conflict of interest. The authors declare no conflicts of interest.

\section{References}

1. Baruah TD. Effectiveness of social media as a tool of communication and its potential for technology enabled connections: a micro-level study. Int J Sci Res 2012;2(5):1-10.

http://www.academia.edu/download/45712283/ijsrpmay-2012-24.pdf.

2. Meskó B. Social Media in Clinical Practice, Springer, London, 2013.

3. Smailhodzic E, Hooijsma W, Boonstra A, Langley DJ. Social media use in healthcare: A systematic review of effects on patients and on their relationship 
with healthcare professionals. BMC Health Serv Res 2016;16:442. https://doi.org/10.1186/s12913-0161691-0.

4. Twitter by the Numbers: Stats, Demographics \& Fun Facts. Available at:

https://www.omnicoreagency.com/twitter-statistics/ accessed 28 August 2018.

5. Pinho-Costa L, Yakubu K, Hoedebecke K, et al. Healthcare hashtag index development: Identifying global impact in social media. J Biomed Inform 2016;63:390-399.

https://doi.org/10.1016/j.jbi.2016.09.010.

6. Winslow CE. The untilled fields of public health. Science. 1920;51(1306):23-33. DOI:

10.1126/science.51.1306.23.

7. WHO. Global and regional estimates of violence against women: prevalence and health effects of intimate partner violence and non-partner sexual violence. Geneva: World Health Organisation, 2013. https://apps.who.int/iris/bitstream/handle/10665/85239 l.

8. Wikipedia contributors, 'Me Too movement', Wikipedia, The Free Encyclopedia, . Available at:

https://en.wikipedia.org/w/index.php?title=Me_Too_m ovement\&oldid=856707128 accessed 27 August 2018.

9. Smith DW, López CM. \#MeToo and Academic Medicine. Fam Med 2018;50(6):423-425. DOI: 10.22454/FamMed.2018.348512.

10. O'Neil A, Sojo V, Fileborn B, Scovelle AJ, Milner A. The \#MeToo movement: an opportunity in public health? Lancet 2018;391(10140):2587-2589. https://doi.org/10.1016/S0140-6736(18)30991-7.

11. Cohen S, Janicki-Daverts D, Miller GE. Psychological stress and disease. JAMA 2007;298(14):1685-1687. DOI:10.1001/jama.298.14.1685

12. Wakefield MA, Loken B, Hornik RC. Use of mass media campaigns to change health behaviour. Lancet 2010;376(9748):1261-1271. https://doi.org/10.1016/S0140-6736(10)60809-4.

13. Randolph W, Viswanath K. Lessons learned from public health mass media campaigns: marketing health in a crowded media world. Annu Rev Public Health 2004;25:419-437. https://doi.org/10.1146/annurev.publhealth.25.101802. 123046.

14. Seymour J. The impact of public health awareness campaigns on the awareness and quality of palliative care. J Pall Med 2018;21(S1):S30-S36. https://doi.org/10.1146/annurev.publhealth.25.101802. 123046.

15. Chang HC. A new perspective on Twitter hashtag use: diffusion of innovation theory. Proc Am Soc Inf Sci Technol 2010;47(1):1-4. https://doi.org/10.1002/meet.14504701295.
16. Moorhead SA, Hazlett DE, Harrison L, et al. A new dimension of health care: systematic review of the uses, benefits, and limitations of social media for health communication. $J$ Med Internet Res 2013;15(4):e85. DOI: 10.2196/jmir.1933.

17. Thackeray R, Crookston BT, West JH. Correlates of health-related social media use among adults. $J$ Med Internet Res 2013;15(1):e21. DOI: 10.2196/jmir.2297.

18. Neiger BL, Thackeray R, Van Wagenen SB, et al. Use of social media in health promotion: purposes, key performance indicators, and evaluation metrics. Health Promot Pract 2012;13(2):159-164. https://doi.org/10.1177/1524839911433467.

19. Eysenbach G. Infodemiology and infoveillance: framework for an emerging set of public health informatics methods to analyze search, communication and publication behavior on the Internet. $J$ Med Internet Res 2009;11(1):e11. DOI: 10.2196/jmir.1157.

20. Shive M, Bhatt M, Cantino A, Kvedar J, Jethwani K. Perspectives on acne: what Twitter can teach health care providers. JAMA Dermatol 2013;149(5):621-622.

21. Cooper CP, Mallon KP, Leadbetter S, Pollack LA, Peipins LA. Cancer Internet search activity on a major search engine, United States 2001-2003. J Med Internet Res 2005;7(3):e36. DOI: $10.2196 /$ jmir.7.3.e36.

22. SalesForce. Available at: https://www.salesforce.com/products/marketingcloud/social-media-marketing/ accessed 28 August 2018.

23. AFDigital. What is Radian6? Available at: http://www.afdigital.com/what-is-radian6/ accessed 28 August 2018.

24. Antman K. Building on \#MeToo to enhance the learning environment for US medical schools. JAMA 2018;319(17):1759-1760.

DOI:10.1001/jama.2018.3812.

25. Monroe KR. Harassment charges: Metoo but due process. Science 2018;361(6403):656.

26. Job and Health Outcomes of Sexual Harassment and How Women Respond to Sexual Harassment. In : Sexual Harassment of Women: Climate, Culture, and Consequences in Academic Sciences, Engineering, and Medicine. Benya FF, Widnall SE, Johnson PA, editors. Washington (DC): National Academies Press (US). 2018. https://www.ncbi.nlm.nih.gov/books/NBK519461.

27. Nielsen M, Einarsen S. Prospective relationships between workplace sexual harassment and psychological distress. Occup Med 2012;62(3):226228. https://doi.org/10.1093/occmed/kqs010.

28. DeGue S, Valle LA, Holt MK, et al. A systematic review of primary prevention strategies for sexual violence perpetration. Aggress Violent Behav 


\section{JOURNAL OF THE INTERNATIONAL SOCIETY FOR TELEMEDICINE AND EHEALTH}

2014;19(4):346-362.

https://doi.org/10.1016/j.avb.2014.05.004. 